\title{
Doenças ocupacionais em profissionais da enfermagem: uma revisão integrativa
}

\author{
Occupational diseases in nursing professionals: an integrating review \\ Enfermedades ocupacionales en profesionales de enfermería: una revisión integrativa \\ João Paulo de Morais PEREIRA 1 \\ Waleska Fernanda Souto NÓBREGA² \\ Ramon Evangelista dos Anjos PAIVA3 \\ ${ }^{1}$ Graduado em Enfermagem, Especialista em Enfermagem do Trabalho, Faculdades Integradas de Patos - FIP, 58416-440 Patos - PB, Brasil \\ ${ }^{2}$ Graduada em Odontologia, Especialista em Saúde Coletiva e da Família, Universidade Estadual da Paraíba, 58429-500 Campina Grande - PB, Brasil \\ ${ }^{3}$ Mestre em Enfermagem, Faculdades Integradas de Patos - FIP, 58416-440 Patos - PB, Brasil
}

\begin{abstract}
Resumo
Objetivo: realizar revisão integrativa da literatura a respeito das doenças ocupacionais mais frequentes dentre os profissionais da Enfermagem. Métodos: A bibliografia foi pesquisada na base de dados Scielo através das palavras chave "doenças ocupacionais" e "enfermagem" com auxílio do boleano "and", onde foram obtidos 255 artigos. Foram selecionados artigos dos últimos 20 anos, onde restaram 78 diretamente relacionados ao tema artigos, dos quais 30 foram selecionados através da leitura do título e resumo e 13 foram efetivamente utilizados para a construção desta revisão, além de 1 documento oficial, totalizando 14 referências. Resultados e Conclusões: Mais estudos são necessários para que se permita refletir e conhecer ainda mais acerca da realidade dos riscos ocupacionais aos que estão expostos os profissionais de saúde, especialmente os trabalhadores da Enfermagem, o que irá contribuir para que a discussão a respeito do tema seja ampliada e se traduza em ações verdadeiramente voltadas para a busca de condições de trabalho dignas para tal categoria, garantindo assim a manutenção da qualidade de vida dos profissionais.
\end{abstract}

Descritores: Doenças Profissionais; Enfermagem; Saúde do Trabalhador.

\section{Abstract}

Objective: to carry out an integrative review of the literature on the most frequent occupational diseases among Nursing professionals. Methods: The bibliography was searched in the Scielo database through the keywords "occupational diseases" and "nursing" with the help of the boolean "and", where 255 articles were obtained. We selected articles from the last 20 years, where 78 were directly related to the theme, of which 30 were selected through the reading of the title and abstract and 13 were effectively used for the construction of this review, in addition to 1 official document, totaling 14 references. Results and Conclusions: More studies are needed to allow us to reflect and know even more about the reality of occupational hazards to which health professionals are exposed, especially Nursing workers, which will contribute to the discussion about the theme be amplified and translated into actions truly aimed at the search for decent working conditions for this category, thus ensuring the maintenance of the quality of life of professionals.

Descriptors: Occupational Diseases; Nursing; Occupational Health.

\section{Resumen}

Objetivo: realizar una revisión integrativa de la literatura acerca de las enfermedades ocupacionales más frecuentes entre los profesionales de la enfermería. Métodos: La bibliografía fue investigada en la base de datos Scielo a través de las palabras clave "enfermedades ocupacionales" y "enfermería" con auxilio del boleano "and", donde se obtuvieron 255 artículos. Se seleccionaron artículos de los últimos 20 años, donde quedaron 78 directamente relacionados al tema artículos, de los cuales 30 fueron seleccionados a través de la lectura del título y resumen y 13 fueron efectivamente utilizados para la construcción de esta revisión, además de 1 documento oficial, totalizando 14 referencias. Resultados y Conclusiones: Más estudios son necesarios para que se permita reflexionar y conocer aún más acerca de la realidad de los riesgos ocupacionales a los que están expuestos los profesionales de salud, especialmente los trabajadores de la Enfermería, lo que contribuirá a que la discusión acerca del tema se amplíe y se traduzca en acciones verdaderamente orientadas a la búsqueda de condiciones de trabajo dignas para dicha categoría, garantizando así el mantenimiento de la calidad de vida de los profesionales.

Descriptores: Enfermedades Profesionales; Enfermería; Salud Laboral.

\section{INTRODUÇÃO}

A lei 8.080 de 19 de Setembro de 1990 apresenta como ações incluídas no campo de ação do Sistema Único de Saúde (SUS) as ações de Saúde do Trabalhador, compreendidas como atividades que se destinam à promoção e proteção da saúde dos trabalhadores, bem como à recuperação e reabilitação da saúde dos trabalhadores submetidos aos riscos e agravos advindos das condições de trabalho'.

Doença ocupacional caracteriza os males que são produzidos ou desencadeados pelo exercício profissional peculiar a determinada atividade. Ainda pode-se afirmar que as doenças ocupacionais são resultados de microtraumas que cotidianamente atingem o trabalhador, e por serem cumulativos, acabam por vencerem suas barreiras de defesas orgânicas ${ }^{2}$.

Os profissionais de enfermagem formam uma parcela considerável do setor profissional, atuando tanto em serviços hospitalares quanto na atenção primária em saúde. São evidenciados constantemente nessa população problemas de saúde como estresse, fadiga e síndrome de bournout. Porém, ainda são poucos os estudos que abordam suas condições laborais e os riscos ocupacionais que estas envolvem, e que possam auxiliar na melhoria das condições de trabalho dos profissionais de enfermagem ${ }^{3}$.

As pesquisas apontam que os profissionais da Enfermagem, devido exercerem uma profissão peculiar em atribuições e local de trabalho, estão diariamente expostos a diversos riscos que podem resultar em doenças laborais.

Neste sentido, o objetivo do presente trabalho é realizar uma revisão integrativa da literatura a respeito das doenças ocupacionais mais frequentemente apresentadas dentre os profissionais da Enfermagem.

\section{MATERIAL E MÉTODO}

$$
\text { Visando atender aos objetivos }
$$

especificados, neste artigo utilizamos o método de revisão integrativa da literatura científica, cuja bibliografia foi pesquisada na base de dados Scielo através das palavras chave "doenças ocupacionais" e 
"enfermagem" com auxílio do boleano "and", onde foram obtidos 255 artigos. Foram selecionados artigos dos últimos 20 anos, onde restaram 78 diretamente relacionados ao tema artigos, dos quais 30 foram selecionados através da leitura do título e resumo e 13 foram efetivamente utilizados para a construção desta revisão, além de 1 documento oficial, totalizando 14 referências.

\section{- Reflexão sobre saúde e dispositivos legais}

Sagre e Ferraz ${ }^{4}$, embora acreditem que o conceito de saúde da Organização Mundial da Saúde (OMS) "situação de perfeito bem-estar físico, mental e social" encontre-se ultrapassada e ainda mais utópica, apontam que saúde caracterizaria um estado de razoável harmonia entre o sujeito e a sua própria realidade. Trazendo aspectos importantes para que se alcance tal estado de saúde, como o bom funcionamento da mente, do corpo, associados ao bom convívio em meio social, inclusive de trabalho.

A Constituição de 1988 trás em seu artigo 196 a saúde é direito de todos, sendo dever do Estado garanti-la por meio de políticas sociais e econômicas que busquem a redução do risco de doença e de outros agravos, bem como o acesso universal e igualitário às ações e serviços para sua promoção, proteção e recuperação ${ }^{1}$.

Complementando o que a Carta Magna traz consigo, a lei 8.080 de 19 de Setembro de 1990, apresenta como ações incluídas no campo de ação do Sistema Único de Saúde (SUS) as ações de Saúde do Trabalhador, compreendidas como atividades que se destinam à promoção e proteção da saúde dos trabalhadores, bem como à recuperação e reabilitação da saúde dos trabalhadores submetidos aos riscos e agravos advindos das condições de trabalho' ${ }^{1}$.

\section{- Doenças ocupacionais}

Doença ocupacional caracteriza os males que são produzidos ou desencadeados pelo exercício profissional peculiar a determinada atividade. Ainda pode-se afirmar que as doenças ocupacionais são resultados de microtraumas que cotidianamente atingem o trabalhador, e por serem cumulativos, acabam por vencerem suas barreiras de defesas orgânicas ${ }^{2}$.

Estudo que aborda a saúde do trabalhador na área da saúde, destaca que a relação entre exposições ocupacionais, compostos químicos e doenças é conhecida há muito tempo e que está inserida no campo saúde-trabalho-ambiente. Porém, traz como fator de vulnerabilidade a pobreza presente na vida de numerosos trabalhadores brasileiros, além da ausência de ações em Saúde do Trabalhador, inclusive as recomentadas pelo Ministério da Saúde (MS), afirmando que apenas a notificação do agravo é realizada, não estando disponíveis ações de prevenção aos riscos e doenças ocupacionais. $\mathrm{O}$ autor ainda salienta um ponto crucial que é a forma como o trabalhador se relaciona com a exposição, como as condições de vida, moradia, acesso a serviços de saúde e educação, nível salarial e escolar ${ }^{5}$.

Silva et al. ${ }^{6}$, esclarecem que ações de saúde do trabalhador, enquanto política pública de saúde, se caracterizam como um campo relativamente novo, com ações muitas vezes desconhecidas pelos próprios trabalhadores. Esse campo, enquanto política pública de saúde, revela-se relativamente novo e, por esse motivo, as ações ainda são muitas vezes desconhecidas pelos profissionais da saúde.

Carvalho et al. ${ }^{5}$ expõem a ideia de que ações de vigilância devem ser constantes nos ambientes de trabalho, a fim de alcançar a melhora nos processos produtivos que torne menos insalubre a atividade do trabalhador, trazendo um bem efetivo para sua realidade social e qualidade de vida.

Magalhães et al. ${ }^{7}$, demonstram em seu estudo que a maioria dos profissionais de enfermagem são do sexo feminino, com média de idade de 40 anos, sedentários, entre outros fatores de risco para doenças ocupacionais, como a Hipertensão Arterial Sistêmica. Os autores deixam claro que tais profissionais tem a necessidade de atenção especial, pois vivem uma profissão estressante, enfrentando problemas como insatisfação profissional, falta de reconhecimento financeiro e algumas doenças ocupacionais, o que pode prejudicar a qualidade de vida e dificultar o autocuidado.

\section{- Os profissionais de enfermagem e as doenças ocupacionais}

Os profissionais de enfermagem formam uma parcela considerável do setor profissional, atuando tanto em serviços hospitalares quanto na atenção primária em saúde. São evidenciados constantemente nessa população problemas de saúde como estresse, fadiga e síndrome de bournout. Porém, ainda são poucos os estudos que abordam suas condições laborais e os riscos ocupacionais que estas envolvem, e que possam auxiliar na melhoria das condições de trabalho dos profissionais de enfermagem ${ }^{3}$.

Mori e Naghettini ${ }^{8}$, investigando a relação saúde do trabalhador - Estratégia de Saúde da Família (ESF) observaram a falta de conhecimento dos próprios profissionais enfermeiros a respeito da área de saúde ocupacional, e também evidenciou que a área ainda é subvalorizada e pouco explorada nos campos acadêmico e profissional, e até mesmo pela gestão de saúde, deixando clara a necessidade de educação permanente na área.

Alguns estudos buscam evidenciar o adoecimento e os riscos ocupacionais de profissionais que atual em hospital, identificando principalmente exposição a bactérias e vírus, tabaco, ruídos, indução a adoção de postura corporal inadequada devido a inadequações ergonômicas e ao estresse. Concluindo que mais da metade dos trabalhadores de saúde apresentam problemas de saúde, embora poucos diagnósticos sejam registrados como doença 
ocupacional ${ }^{9}$.

Estudo de Nazário et al. ${ }^{10}$, que teve como propósito investigar trabalhadores de enfermagem da terapia intensiva, observou que os riscos ergonômicos, psicológicos ou emocionais, químicos e biológicos foram os mais frequentes no ambiente de trabalho, os quais foram considerados inerentes à profissão. Nazário ainda destaca que a prevenção de riscos e doenças ocupacionais é possível e precisa ser incentivada.

Pesquisa buscando investigar doenças ocupacionais em profissionais da enfermagem em hospitais e Unidades Básicas de Saúde (UBS) evidenciaram uma alta prevalência de síndrome de Burnout, trazendo como fatores de risco mais prevalentes problemas de relacionamento com colegas de trabalho e insatisfação com o trabalho. Deixando perceptível a realização de mais pesquisas nessa área, tendo em vista a complexidade do trabalho em enfermagem e as diferentes situações em que os profissionais atuam ${ }^{11}$.

Em seu estudo, Shoji et al. ${ }^{12}$ afirmaram estar presentes no ambiente de trabalho da enfermagem os riscos físicos, químicos, biológicos, ergonômicos e de acidentes, que na maioria das vezes decorrem das inadequações da estrutura, dos equipamentos, do espaço físico e do quantitativo de trabalhadores das equipes de enfermagem. Cabendo a Enfermagem, cumprir o desafio de reorientar sua prática, transformar seu processo de trabalho afim de minimizar o impacto da sua divisão social e da sua organização, a fim de não apresentar repercussões negativas física e psiquicamente nos trabalhadores. Pois apenas assim o profissional trabalhará com dignidade e segurança, conforme priorizado pela política de saúde do trabalhador.

\section{RESULTADOS E DISCUSSÃO}

Segundo Silva e Marziale ${ }^{14}$, a maior frequência de problema de saúde que assola os enfermeiros está relacionada ao sistema respiratório, geniturinário, problemas relativos aos órgãos dos sentidos, sistema digestivo, ósteomuscular ou aparelho reprodutor feminino. O que Narzário et al. ${ }^{10}$ explicam como sendo provenientes dos riscos riscos ergonômicos, psicológicos ou emocionais, químicos e biológicos inerentes à profissão.

Dentre os riscos ocupacionais mais frequentemente identificados, os psicossociais foram os mais citados no estudo de Dalri et al. ${ }^{13} \mathrm{e}$ as alterações de saúde mais encontradas foram, algias, cansaço mental e estresse, distúrbios do sono, alterações cardiovasculares e processos infecciosos, corroborando com Magalhães et al. ${ }^{7}$, que afirmam que tais problemas ocorrem devido as pessoas viverem uma profissão estressante, enfrentarem problemas como insatisfação profissional e falta de reconhecimento financeiro, o que pode prejudicar a qualidade de vida e dificultar o autocuidado.

Silva e Marziale $^{14}$ apontam em seu estudo alguns dos problemas de saúde que podem estar relacionados às condições de trabalho e ambiente laboral peculiares da enfermagem, indicando a necessidade de uma análise aprofundada e diferenciada dos diversos locais de trabalho. Tal afirmação corrobora com Shoji et al. ${ }^{12}$ que discorrem sobre diversos dos riscos da profissão estarem relacionados a inadequações da estrutura, dos equipamentos, do espaço físico e do quantitativo de trabalhadores das equipes de enfermagem.

\section{CONCLUSÃO}

As pesquisas apontam que os profissionais da Enfermagem, devido exercerem uma profissão peculiar em atribuições e local de trabalho, estão diariamente expostos a diversos riscos que podem resultar em doenças laborais.

Faz-se necessário que se desenvolvam políticas de educação permanente para informar os próprios profissionais como evitar tais riscos, bem como é de extrema importância a inspeção periódica do local de trabalho.

Mais estudos são necessários para que se permita refletir e conhecer ainda mais acerca da realidade dos riscos ocupacionais aos que estão expostos os profissionais de saúde, especialmente os trabalhadores da Enfermagem, o que irá contribuir para que a discussão a respeito do tema seja ampliada e se traduza em ações verdadeiramente voltadas para a busca de condições de trabalho dignas para tal categoria, garantindo assim a manutenção da qualidade de vida dos profissionais.

\section{REFERÊNCIAS}

1. Brasil. Doença ocupacional. Brasília : Senado Federal, Coordenação de Edições Técnicas, 2016.

2. Monteiro AL, Bertagni FS. Acidentes de trabalho e doenças ocupacionais. 8 ed., São Paulo: Saraiva, 2016.

3. Canales-Vergara M, Valenzuela-Suazo S, ParavicKlijn T. Condiciones de trabajo de los profesionales de enfermería en Chile M. Enferm univ. 2016;13(3):178-86.

4. Segre M, Ferraz FC. O conceito de saúde. Rev. Saúde Pública. 1997;31(5):538-42.

5. Carvalho LVB, Costa-Amaral IC, Mattos RCOC, Larentis AL. Exposição ocupacional a substâncias químicas, fatores socioeconômicos e Saúde do Trabalhador: uma visão integrada. Saúde debate. 2017;41(Esp):313-26.

6. Silva A, Ferraz L, Rodrigues-Júnior SA. Ações em Saúde do Trabalhador desenvolvidas na Atenção Primária no município de Chapecó, Santa Catarina Rev bras saude ocup. 2016;41:e16.

7. Magalhães FJ, Mendonça LBA, Rebouças CBA, Lima FET, Custodio IL, Oliveira SC. Fatores de 
risco para doenças cardiovasculares em profissionais de enfermagem: estratégias de promoção da saúde, Rev Bras Enferm. 2014;67(3):394-400.

8. Mori EC, Naghettini AV. Formação de médicos e enfermeiros da estratégia Saúde da Família no aspecto da saúde do trabalhador Rev Esc Enferm USP · 2016;50(spe):25-31

9. Fernandes MA, Marziale MHP. Riscos ocupacionais e adoecimento de trabalhadores em saúde mental. Acta paul enferm. 2014; 27(6):539-47.

10.Nazario EG, Camponogara S, Dias GL. Riscos ocupacionais e adesão a precauções-padrão no trabalho de enfermagem em terapia intensiva: percepções de trabalhadores. Rev bras saude ocup. 2017;42:e7

11.Campos ICM, Angélicos AP, Oliveira MS, Oliveira DCR. Fatores Sociodemográficos e Ocupacionais Associados à Síndrome de Burnout em Profissionais de Enfermagem. Psicol Reflex Crit. 2015;28(4):764-71.

12.Shoji S, Souza NVDO, Farias SNP, Vieira MLC, Progianti JM. Proposta de melhoria das condições de trabalho em uma unidade ambulatorial: perspectiva da enfermagem Esc Anna Nery. 2016;20(2):303-9.

13.Dalri RCMB, Robazzi MLCC, Silva LA. Riscos ocupacionais e alterações de saúde entre trabalhadores de enfermagem brasileiros de unidades de urgência e emergência. Cienc enferm. 2010;16(2):69-81.

14. Silva DMPP, Marziale MHP. Condições de trabalho versus absenteísmo-doença no trabalho de enfermagem. Ciênc cuid saúde. 2006;5(suppl):166-72.

\section{CONFLITO DE INTERESSES}

Os autores declaram não haver conflitos de interesse.

\section{AUTOR PARA CORRESPONDENCIA}

Waleska Fernanda Souto Nóbrega

drawaleskasouto@gmail.com

Submetido em 09/01/2019

Aceito em 12/03/2019 